\title{
Estudo da coagulação/floculação de água com turbidez moderada na sedimentação e flotação por ar dissolvido
}

\author{
Study of coagulation/flocculation of water with moderate \\ turbidity in sedimentation and floating by dissolved air \\ Verônica dos Santos Lopes ${ }^{1} \oplus$, Lorena Miranda de Almeida Silva' ${ }^{\circledR}$, \\ Rodrigo Braga Moruzzi ${ }^{2} \odot$, André Luiz de Oliveira' ${ }^{\circledR}$
}

\section{RESUMO}

Os processos de formação, crescimento, ruptura e rearranjo dos flocos que ocorrem durante a coagulação/floculação influenciam, significativamente, a eficiência da remoção dos agregados no tratamento de água. Por isso, é importante estudar as propriedades dos flocos, que são alteradas continuamente durante a floculação e podem interferir na subsequente remoção das partículas. Neste trabalho foi avaliada a eficiência de remoção de turbidez por meio da sedimentação e da flotação por ar dissolvido (FAD), com o uso de aluminato de sódio como agente coagulante. Adicionalmente, a etapa de floculação foi monitorada usando imagens obtidas por método não intrusivo. A FAD proporcionou melhor resultado de remoção de turbidez, de 93\%, mostrando robustez como etapa no processo de tratamento de água. Na etapa de floculação, o tamanho dos flocos variou de 0,03 a 0,53 mm, com maior concentração de partículas nas menores classes de tamanho monitoradas. O estudo da dimensão fractal mostrou que o aumento da intensidade da mistura acentuou a ruptura $\left(D_{\text {médio }}\right.$ de $0,2 \mathrm{~mm}$ para $20 \mathrm{~s}^{-1}$ para $\mathrm{D}_{\text {médio }}$ de $0,1 \mathrm{~mm}$ para $40 \mathrm{~s}^{-1}$ ) nos flocos, promovendo o surgimento de flocos menos compactos. A FAD se mostrou menos sensível às variações de tamanho dos agregados quando comparada à sedimentação.

Palavras-chave: floculação; sedimentação; flotação por ar dissolvido; propriedades dos flocos.

\begin{abstract}
The processes of formation, growth, rupture, and rearrangement of flocs, which occur during coagulation/flocculation, significantly influence the removal efficiency of the aggregates in water treatment. Therefore, it is important to study the flocs properties because they change continuously during the process and may interfere in the choice of later step for removal of the particles. This article evaluated the efficiency of turbidity removal in sedimentation and dissolved air flotation (DAF) with the use of sodium aluminate as a coagulant. In addition, the flocculation step was monitored through digital images, by non-intrusive method, to study the morphological properties of flocs. The DAF step provided better removal results (greater than 93\%) in less process time, showing to be an advantageous step in turbid water treatment. In the flocculation step, the size of the flocs varied from 0.03 to $0.53 \mathrm{~mm}$, with a higher particle concentration in the smaller size classes. The study of the fractal dimension showed that the increase in the intensity of the mixture accentuated the rupture ( $D_{\text {mean }}$ of $0.2 \mathrm{~mm}$ for 2Osfor $\mathrm{D}_{\text {mean }}$ of $\mathrm{O} .1 \mathrm{~mm}$ for $4 \mathrm{Os}^{-1}$ ) in the flocs, promoting the appearance of less compact flocs. DAF was less sensitive to variations in aggregate size when compared to sedimentation.
\end{abstract}

Keywords: flocculation; sedimentation; dissolved air flotation; flocs properties.

\section{INTRODUÇÃO}

A água pode conter uma variedade de impurezas que apresentam carga superficial negativa, impedindo que se aproximem umas das outras e fazendo com que permaneçam inalteradas no meio. Para que essas impurezas sejam removidas, as partículas presentes na água devem ser desestabilizadas, no caso de estações de tratamento de água (ETA), na etapa de coagulação, após a adição de coagulante e intensa agitação. Na floculação, o choque entre as partículas desestabilizadas ocorre por meio de agitação lenta, formando flocos que são subsequentemente removidos nas etapas seguintes, como sedimentação ou flotação e filtração.

O estudo das condições operacionais da coagulação e floculação, como o tipo e a dosagem de coagulante, a intensidade da mistura e 
o valor do $\mathrm{pH}$ de coagulação, é necessário para melhorar a eficiência do processo (REN et al., 2017). Além disso, as características dos flocos, tais como tamanho, estrutura e resistência, mudam durante a floculação, com tendência de estabilização, e exercem papel importante na escolha da próxima etapa do tratamento de água (NASSER, 2014; VANDAMME et al., 2014; REN et al., 2017; MORUZZI et al., 2017; MORUZZI et al., 2019). Para o estudo das características dos flocos, determinantes no processo de separação, podem ser utilizadas técnicas de análise de imagens não intrusivas, que permitem o acompanhamento da evolução da distribuição de tamanho das partículas (DTP) e da estrutura morfológica dos flocos durante o processo de floculação (MORUZZI; REALI, 2007; BUSHELL et al., 2002).

A sedimentação é uma operação unitária de separação sólido-líquido utilizada para a remoção de partículas discretas e floculentas, como sólidos suspensos, partículas coloidais e compostos orgânicos (WANG et al., 2011; HUA et al., 2016). A flotação por ar dissolvido (FAD) geralmente é uma alternativa à sedimentação, pois, com instalações mais compactas, pode permitir a mesma eficiência de remoção. Entretanto a FAD não se limita a águas com partículas de baixa densidade e pode ser empregada até mesmo naquelas com picos de turbidez (REALI; MORUZZI; PATRIZZI, 2003). Na FAD, a ascensão de partículas previamente floculadas ocorre com a introdução de água saturada com ar na base da unidade de tratamento, que à pressão próxima da atmosférica precipita microbolhas que se aderem aos flocos, diminuindo sua densidade efetiva. Esses agregados, formados pela união da bolha de ar e floco, são conduzidos à superfície da unidade de flotação, de onde são removidos (REALI, 1991; MORUZZI; REALI, 2014; YUHENG et al., 2011; BONDELIND; SASIC; BERGDAHL, 2013).

As etapas de coagulação e floculação, que ocorrem antes da sedimentação e da FAD, afetam diretamente o desempenho das unidades de separação subsequentes no tratamento de água. Por essa razão, é fundamental avaliar as condições de mistura e as características dos flocos para a escolha adequada da etapa subsequente e, assim, melhorar a eficiência de remoção. O presente trabalho teve como objetivo verificar a sensibilidade da sedimentação e da FAD para pequenas alterações de tamanho dos flocos formados durante a floculação.

\section{MATERIAIS E MÉTODOS}

O aluminato de sódio foi utilizado como coagulante químico, preparando-se uma solução com concentração de 20 g.L $\mathrm{L}^{-1}$, de modo que cada $1 \mathrm{~mL}$ de solução contivesse $20 \mathrm{mg}$ de aluminato de sódio. Para conferir turbidez à água de estudo, foi empregada suspensão de caulinita da marca Sigma-Aldrich, preparada de acordo com a metodologia adotada por Yukselen e Gregory (2004). Para ajuste do pH da água de estudo foi utilizada solução estoque de $\mathrm{HCl}$ com concentração de
0,5 M. A água de estudo foi preparada usando água destilada e solução estoque de caulinita para turbidez de $50 \pm 2$ em Nephelometric Turbidity Unit (NTU).

Os ensaios de coagulação/floculação foram conduzidos em reatores estáticos de bancada. Os experimentos foram realizados como se segue: agitação rápida inicial a $900 \mathrm{~s}^{-1}$ durante $10 \mathrm{~s}$ após a adição da solução para ajustar o pH e depois $10 \mathrm{~s}$ após a adição do coagulante, seguida por floculação durante $20 \mathrm{~min}$. Os gradientes médios de velocidade da floculação avaliados foram de 20, 30 e $40 \mathrm{~s}^{-1}$. Ao término do tempo de floculação de $20 \mathrm{~min}$, foram conduzidos os ensaios de sedimentação e FAD. Para os ensaios de sedimentação, as velocidades de sedimentação foram de 5, 2,5,1 e 0,5 cm.min ${ }^{-1}$. Os ensaios de FAD foram realizados em um equipamento de flotação de bancada, com produção de água saturada com ar em uma câmara de saturação a 5 Bar durante 10 min. As taxas de recirculação foram de 5, 10 e 15\%, e as velocidades ascensionais foram de 24, 12 e $6 \mathrm{~cm} \cdot \mathrm{min}^{-1}$.

A eficiência da remoção de turbidez foi calculada de acordo com a Equação 1:

$T(\%)=\left(1-\frac{T_{e}}{T_{i}}\right) \times 100$

Em que:

$T_{\mathrm{i}}=$ a turbidez inicial da água de estudo antes da coagulação;

$T_{\mathrm{e}}=$ a turbidez da água ao fim das etapas de sedimentação e FAD nas diferentes velocidades de sedimentação e flotação, respectivamente.

Os flocos foram monitorados durante os ensaios de coagulação/floculação usando procedimentos de aquisição de imagens, de acordo com os métodos descritos por Moruzzi e Reali (2007), Oliveira et al. (2015), Moruzzi et al. (2017) e Oliveira et al. (2018). No presente trabalho as imagens foram obtidas por câmera digital (Nikon D5500), com resolução de $6.000 \times 4.000$ pixels, a partir de plano de luz gerado por uma fonte de laser com potência nominal de $98.000 \mathrm{~mW}$ e comprimento de onda verde de 532 nm, como mostrado na Figura 1.

A aquisição das imagens dos flocos ocorreu para os tempos de floculação $\left(T_{\mathrm{f}}\right)$ de 1, 3, 5, 10, 15 e $20 \mathrm{~min}$. Para cada $T_{\mathrm{f}}, 40$ imagens foram obtidas à frequência de quatro imagens por segundo, acionando-se o disparo da câmera fotográfica por meio do programa de gerenciamento CPP 2.0, o que totalizou 240 imagens para cada $G_{f}$ avaliado. $O$ tratamento das imagens foi realizado pelo programa computacional de uso livre ImageJ 1.51, utilizado para a caracterização dos flocos formados durante a floculação.

Neste trabalho, o parâmetro de forma avaliado foi a dimensão fractal 2D, definida, em termos bidimensionais, pela relação potencial entre a área de uma partícula $\left(A_{\mathrm{s}}\right)$, seu comprimento característico $(l)$ e a dimensão da área fractal $\left(D_{\mathrm{f}}\right)$, conforme Equação 2. 


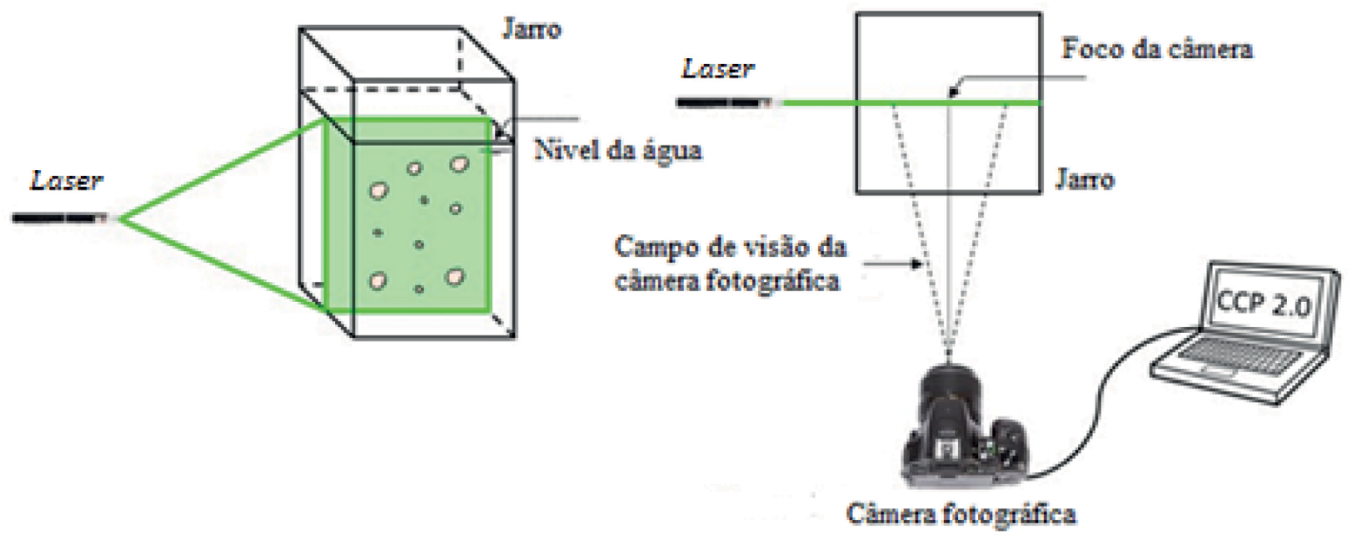

Fonte: Oliveira et al. (2018).

Figura 1 - Aparato experimental utilizado para a aquisição de imagens durante a floculação: vista frontal (à esquerda) e vista superior (à direita).

$A_{S} \sim l^{D_{f}}$

A dimensão fractal calculada para toda a população de flocos é representada pelo coeficiente de inclinação da reta que melhor se ajusta à distribuição dos dados. Para objetos com geometria euclidiana, $D_{\mathrm{f}}$ é um número inteiro, mas para objetos fractais apresenta valores fracionários entre um e dois no espaço bidimensional.

A análise da DTP foi realizada com base no diâmetro médio equivalente em área das partículas, calculado de acordo com a Equação 3.

$D_{m e ́ d}=\sqrt{\frac{4 \cdot A}{\pi}}$

Em que:

$D_{\text {méd }}=$ diâmetro médio $(\mathrm{mm})$;

$A=$ área média do floco $\left(\mathrm{mm}^{2}\right)$.

O número de flocos e o $D_{\text {méd }}$ foram obtidos para cada $T_{\mathrm{f}}$ nos diferentes $G_{\mathrm{f}}$, e dessa forma ordenados em classes de tamanho.

\section{RESULTADOS E DISCUSSÃO}

Conforme mencionado, foram realizados ensaios de sedimentação e flotação para avaliar a eficiência de remoção de turbidez. A Tabela 1 mostra os valores de remoção de turbidez em porcentagem, na FAD, para as taxas de recirculação de 5, 10 e 15\% e os gradientes médios de velocidade de 20, 30 e $40 \mathrm{~s}^{-1}$. A velocidade de flotação $\left(\mathrm{V}_{\mathrm{f}}\right)$ que proporcionou maior remoção de turbidez foi $6 \mathrm{~cm} / \mathrm{min}$, com remoção superior a $93 \%$ para todos os $G_{\mathrm{f}}$ avaliados.
Tabela 1 - Valores de remoção de turbidez (\%) na flotação por ar dissolvido para as condições estudadas

\begin{tabular}{c|c|c|c}
\multicolumn{5}{|c}{$20 \mathrm{~s}^{-1}$} \\
\hline Taxa de recirculação & $24 \mathrm{~cm} \cdot \mathrm{min}^{-1}$ & $12 \mathrm{~cm} \cdot \mathrm{min}^{-1}$ & $6 \mathrm{~cm} \cdot \mathrm{min}^{-1}$ \\
\hline $5 \%$ & 62,2 & 90,4 & 96,2 \\
\hline $10 \%$ & 45,8 & 94,4 & 97,6 \\
\hline $15 \%$ & 50,0 & 94,2 & 96,4 \\
\hline \multicolumn{4}{|c|}{$30 \mathrm{~s}^{-1}$} \\
\hline Taxa de recirculação & $24{\mathrm{~cm} \cdot \mathrm{min}^{-1}}^{-1}$ & $12 \mathrm{~cm} \cdot \mathrm{min}^{-1}$ & $6 \mathrm{~cm} \cdot \mathrm{min}^{-1}$ \\
\hline $5 \%$ & 71,6 & 92,8 & 95,2 \\
\hline $10 \%$ & 41,0 & 73,4 & 93,0 \\
\hline $15 \%$ & 76,4 & 94,8 & 96,0 \\
\hline \multicolumn{4}{|c|}{$40 \mathrm{~s}^{-1}$} \\
\hline Taxa de recirculação & $24 \mathrm{~cm} \cdot \mathrm{min}^{-1}$ & $12 \mathrm{~cm} \cdot \mathrm{min}^{-1}$ & $6 \mathrm{~cm} \cdot \mathrm{min}^{-1}$ \\
\hline $5 \%$ & 49,0 & 84,2 & 95,8 \\
\hline $10 \%$ & 51,0 & 91,0 & 96,2 \\
\hline $15 \%$ & 64,6 & 90,6 & 93,2 \\
\hline
\end{tabular}

Na Tabela 2 são apresentados os valores percentuais de remoção de turbidez considerando as velocidades de sedimentação e gradientes de floculação estudados. A maior remoção foi observada para o $G_{\mathrm{f}}$ de $20 \mathrm{~s}^{-1}$, de $96,8 \%$, para a velocidade de sedimentação de $0,5 \mathrm{~cm} \cdot \mathrm{min}^{-1}$.

Verifica-se que tanto a sedimentação quanto a flotação foram eficazes na remoção de material suspenso, monitorado indiretamente por meio da turbidez, no entanto a remoção via FAD ocorreu em maiores velocidades, o que em tese resultaria no emprego de maiores taxas de aplicação em unidades em escala plena. 
Na Figura 2 são mostradas a evolução da DTP em função do tempo

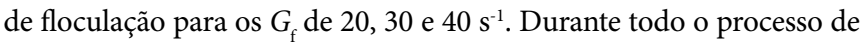
floculação, observou-se maior quantidade de flocos na primeira classe de tamanho $(0,03$ a $0,11 \mathrm{~mm})$, sendo a frequência de partículas pequenas $(0,03$ a $0,09 \mathrm{~mm})$ maior para o $G_{\mathrm{f}}$ de $40 \mathrm{~s}^{-1} \mathrm{em}$ comparação com os

Tabela 2 - Valores de remoção de turbidez (\%) para diferentes velocidades de sedimentação.

\begin{tabular}{|c|c|c|c|}
\hline \multicolumn{5}{|c|}{$20 \mathrm{~s}^{-1}$} \\
\hline $5,0 \mathrm{~cm} \cdot \mathrm{min}^{-1}$ & $2,5 \mathrm{~cm} \cdot \mathrm{min}^{-1}$ & $1,0 \mathrm{~cm} \cdot \mathrm{min}^{-1}$ & $0,5 \mathrm{~cm} \cdot \mathrm{min}^{-1}$ \\
\hline 56,8 & 87,6 & 92,9 & 96,8 \\
\hline $5,0 \mathrm{~cm} \cdot \mathrm{min}^{-1}$ & $2,5 \mathrm{~cm} \cdot \mathrm{min}^{-1}$ & $1,0 \mathrm{~cm} \cdot \mathrm{min}^{-1}$ & $0,5 \mathrm{~cm} \cdot \mathrm{min}^{-1}$ \\
\hline 25,6 & 82,6 & 91,3 & 95,7 \\
\hline \multicolumn{4}{|c|}{$40 \mathrm{~s}^{-1}$} \\
\hline $5,0 \mathrm{~cm} \cdot \mathrm{min}^{-1}$ & $2,5 \mathrm{~cm} \cdot \mathrm{min}^{-1}$ & $1,0 \mathrm{~cm} \cdot \mathrm{min}^{-1}$ & $0,5 \mathrm{~cm} \cdot \mathrm{min}^{-1}$ \\
\hline 3,0 & 48,6 & 84,5 & 92,9 \\
\hline
\end{tabular}

$G_{\mathrm{f}}$ de 20 e $30 \mathrm{~s}^{-1}$, em virtude do aumento da dissipação de energia, que desloca o equilíbrio de agregação e ruptura dos flocos no sentido da componente de ruptura. Isso ocasionou, após a agregação dos flocos nos primeiros minutos, o aumento do número de menores partículas $\mathrm{e}$ a consequente diminuição do número de partículas maiores, enquanto para o $G_{\mathrm{f}}$ de $20 \mathrm{~s}^{-1}$ a quantidade de partículas nas maiores faixas $(0,47$ a $0,53 \mathrm{~mm}$ ) foi maior comparada às dos demais gradientes médios de velocidade de floculação. Observa-se que a DTP para o $G_{\mathrm{f}}$ de $30 \mathrm{~s}^{-1}$ mostra comportamento intermediário em relação aos $G_{\mathrm{f}}$ de $20 \mathrm{~s}^{-1} \mathrm{e}$ de $40 \mathrm{~s}^{-1}$.

A translação para as maiores classes é mais acentuada para o $G_{f}$ de $20 \mathrm{~s}^{-1}$, tendo o $\mathrm{G}_{\mathrm{f}}$ de $40 \mathrm{~s}^{-1}$ promovido recuperação da frequência da menor classe com o tempo.

Yao, Nan e Chen (2014) avaliaram o efeito da turbidez da água de estudo no desenvolvimento da DTP durante a floculação. O estudo foi conduzido com águas preparadas à base de caulinita, com valores de turbidez de 100, 80 e 40 NTU, e 2 mg.L-1 de ácido húmico. A dosagem de coagulante foi de $30 \mathrm{mg} . \mathrm{L}^{-1}$ de cloreto de polialumínio.
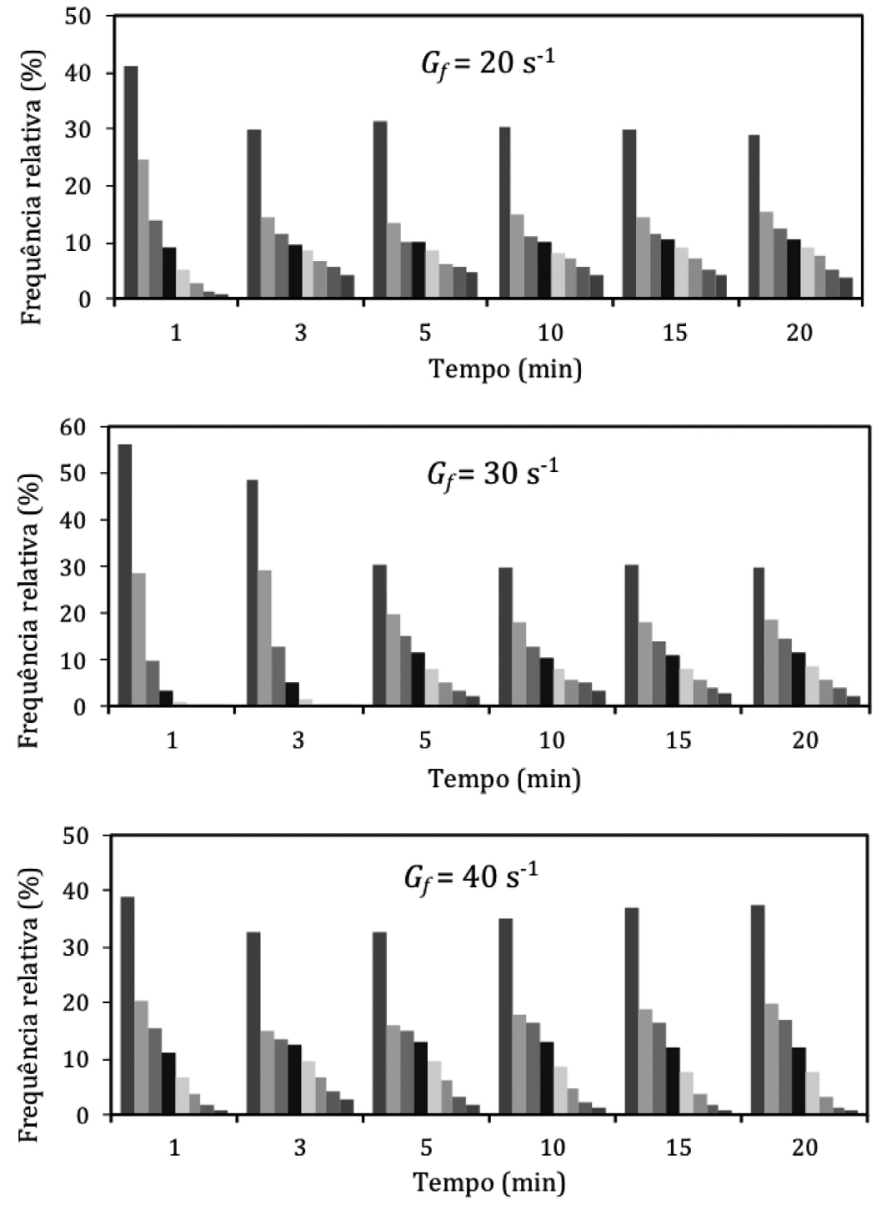

$=0,04-0,10$

민 $0,10-0,16$

$=0,16-0,22$

- $0,22-0,28$

$=0,28-0,35$

$=0,35-0,41$

= $0,41-0,47$

- 0,47-0,53

Faixas de tamanho (mm)

- 0,05-0,11

I $0,11-0,16$

- $0,16-0,22$

- $0,22-0,28$

$0,28-0,34$

$=0,34-0,40$

- $0,40-0,46$

- $0,46-0,52$

Faixas de tamanho (mm)

- $0,03-0,09$

= $0,09-0,14$

= $0,14-0,20$

- $0,20-0,26$

0,26-0,32

$=0,32-0,37$

- $0,37-0,43$

- $0,43-0,49$

Faixas de tamanho $(\mathrm{mm})$

Figura 2 - Evolução da distribuição de tamanho das partículas ao longo do tempo de floculação, dividida em faixas de tamanho (mm), nos gradientes de velocidade de 20,30 e $40 \mathrm{~s}^{-1}$. 
Com o aumento do tempo de floculação, o número de partículas em todas as condições estudadas pelos autores atingiu um valor limite e com pouca variação, o que implica, assim como observado nesse artigo, o equilíbrio entre agregação e ruptura dos flocos.

$\mathrm{Na}$ Figura 3 são mostradas a evolução temporal da dimensão fractal $\left(D_{\mathrm{f}}\right)$ e do diâmetro médio equivalente $\left(D_{\text {eq }}\right)$ para os $G_{\mathrm{f}}$ de 20,30 e $40 \mathrm{~s}^{-1}$ no desenvolvimento do tempo de floculação. Pode-se observar que os valores de $D_{\mathrm{f}}$ variaram de 1,55 a 1,95 , concordando com os valores observados nos trabalhos de Sun et al. (2013) e Moruzzi et al. (2017). Os valores de $D_{\mathrm{f}}$ decresceram com o aumento de $T_{\mathrm{f}}$ de 5 min de floculação em diante, e para o $G_{\mathrm{f}}$ de $40 \mathrm{~s}^{-1}$ a variação de $D_{\mathrm{f}}$ ao longo do tempo foi maior, comprovando que a morfologia do floco é alterada pela energia dissipada no meio. Conforme Xu et al. (2016) e Moruzzi et al. (2017), o aumento de $G_{\mathrm{f}}$ (e consequentemente da dissipação de energia) proporcionou flocos com morfologia mais distante da esfera perfeita, como se observa na Figura 3.

A evolução do diâmetro médio equivalente $\left(D_{\text {méd }}\right)$ foi semelhante para os três $G_{\mathrm{f}}$ avaliados. Verifica-se que a formação de flocos maiores foi favorecida pelo $G_{\mathrm{f}}$ de $20 \mathrm{~s}^{-1}$ e o aumento do gradiente médio de floculação promoveu a ruptura dos flocos e diminuição do $D_{\text {méd }}$, como também observado por Wang et al. (2011) e Moruzzi et al. (2017).

O tamanho e a forma dos agregados de fractal são dependentes das etapas de coagulação e floculação e constituem variáveis importantes na separação de material suspenso por técnicas de clarificação. Os resultados apresentados neste artigo mostram que a FAD pode ser mais robusta que a sedimentação e menos suscetível às alterações dos agregados formados. Evidentemente, essa constatação preliminar carece de mais investigação, visando ampliar os resultados e consolidar o entendimento.

\section{CONCLUSÕES}

O diâmetro dos flocos variou de 0,03 a $0,53 \mathrm{~mm}$, com maior concentração de partículas nas menores classes de tamanho, considerando-se o tempo de desenvolvimento da floculação. Os resultados obtidos para a dimensão fractal indicam que o aumento da intensidade de mistura intensifica a ruptura

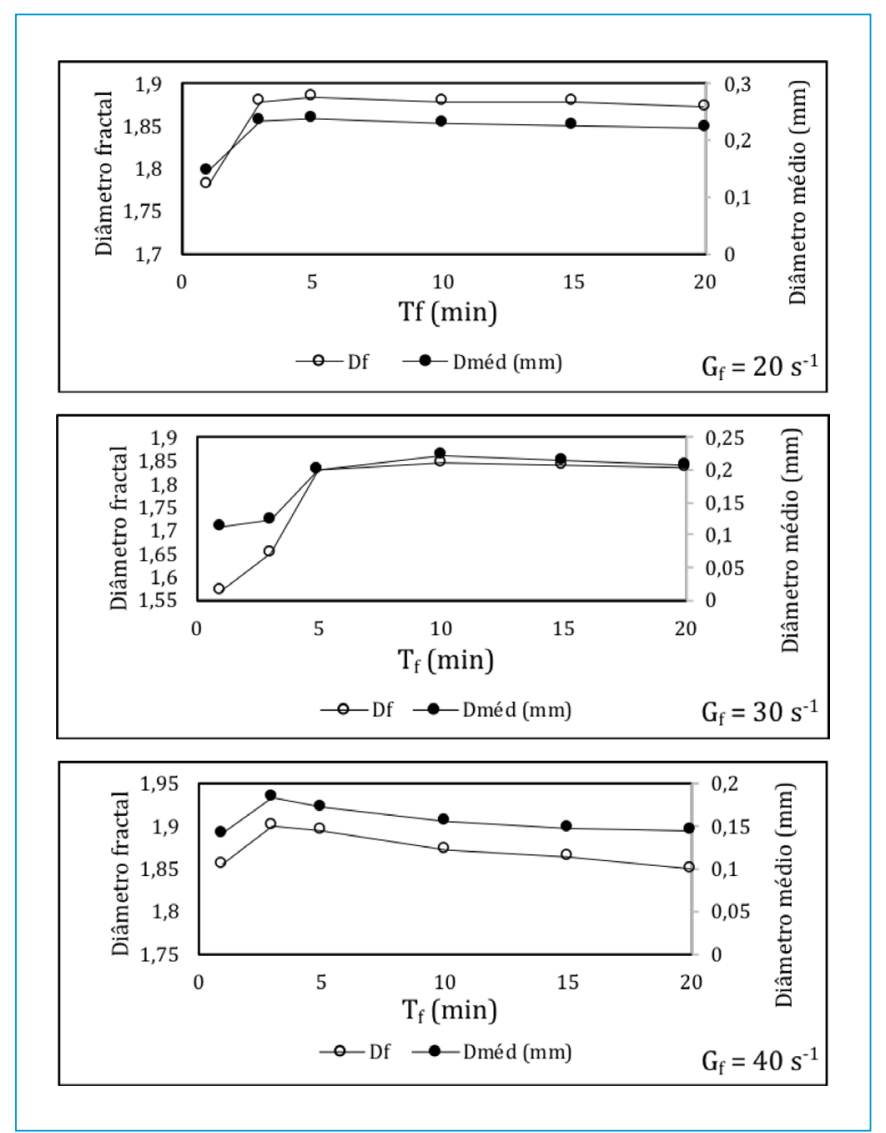

Figura 3 - Evolução da dimensão fractal $\left(D_{f}\right)$ e do diâmetro médio equivalente ao longo do tempo de floculação $\left(T_{f}\right)$, nos gradientes de velocidade de 20,30 e $40 \mathrm{~s}^{-1}$.

e o surgimento de flocos menores e menos compactos, os quais podem favorecer a FAD. Os resultados indicam que a FAD é menos sensível às alterações do gradiente médio de velocidade da floculação.

\section{AGRADECIMENTOS}

Os autores agradecem à Coordenação de Aperfeiçoamento de Pessoal de Nível Superior (CAPES), à Fundação de Amparo à Pesquisa do Estado de Minas Gerais (FAPEMIG) - Processo APQ-01679-17 - e à Faculdade de Engenharia Civil da Universidade Federal de Uberlândia (UFU), a infraestrutura disponibilizada.

\section{REFERÊNCIAS}

BONDELIND, M.; SASIC, S.; BERGDAHL, L. (2013) A model to estimate the size of aggregates formed in a Dissolved Air Flotationunit. Applied Mathematical Modelling, v. 37, n. 5, p. 3036-3047. https://doi. org/10.1016/j.apm.2012.07.004
BUSHELL, G.; YAN, Y.D.; WOODFIELD, D.; RAPER, J.; AMAL, R. (2002) On techniques for the measurement of the mass fractal dimension of aggregates. Advances in Colloid and Interface Science, v. 95, n. 1, p. 1-50. https://doi.org/10.1016/S0001-8686(00)00078-6 
HUA, B.; XIONG, H.; ZHU, G.; WANG, L.; YAN, S.; YANG, J.; DENG, B. (2016) Physico-Chemical Processes. Water Environment Research, v. 88, n. 10, p. 966-1000. https://onlinelibrary.wiley.com/doi/epdf/10.2 175/106143016×14696400494452

MORUZZI, R.B.; OLIVEIRA, A.L.; CONCEIÇÃO, F.T.; GREGORY, J.; CAMPOS, L.C. (2017) Fractal dimension of large aggregates under different flocculation conditions. Science of the Total Environment, v. 609, p. 807-814. https://doi.org/10.1016/j.scitotenv.2017.07.194

MORUZZI, R.B.; REALI, M.A.P. (2007) Método para determinação de distribuição de tamanho de microbolhas (DTMB) em sistemas flotação (FAD) para tratamento de águas utilizando a análise de imagem digital. Engenharia Sanitária e Ambiental, v. 12, n. 3, p. $273-$ 283. https://doi.org/10.1590/S1413-41522007000300007

MORUZZI, R.B.; REALI, M.A.P. (2014) The influence of floc size and hydraulic detention time on the performance of a dissolved air flotation (DAF) pilot unit in the light of a mathematical model. Bioprocess and Biosystems Engineering, v. 37, n. 12, p. 2445-2452. https://orcid.org/0000-0002-1573-3747

MORUZZI, R.B.; SILVA, P.G.; SHARIFI, S.; CAMPOS, L.C.; GREGORY, J. (2019) Strength assessment of alhumic and Al-Kaolin aggregates by intrusive and non-intrusive methods. Separation and Purification Technology, v. 217, p. 265-273. https://doi.org/10.1016/j. seppur.2019.02.033

NASSER, M.S. (2014) Characterization of floc size and effective floc density of industrial paper making suspensions. Separation and Purification Technology, v. 122, p. 495-505. https://doi.org/10.1016/j. seppur.2013.12.008

OLIVEIRA, A.S.; LOPES, V.S.; COUTINHO FILHO, U.; MORUZZI, R.B.; OLIVEIRA, A.L. (2018) Neural network for fractal dimension evolution. Water Science \& Technology, v. 78, n. 4, p. 795-802. http:// dx.doi.org/10.2166/wst.2018.349

OLIVEIRA, A.L.; MORENO, P.; SILVA, P.A.G.; DE JULIO, M.; MORUZZI, R.B. (2016) The effect of form and particle size distribution (PSD) on the removal of particulate matter. Desalination and Water Treatment, v. 57 , n. 36, p. 16721-16732. https://doi.org/10.1080/19443994.2015.1081833

REALI, M.A.P. Concepção e avaliação de um sistema compacto para tratamento de águas de abastecimento utilizando o processo de flotação por ar dissolvido e filtração com taxa declinante. $373 f$. Tese (Doutorado em Engenharia Civil) - Escola de Engenharia de São Carlos, Universidade de São Paulo, São Carlos, 1991.
REALI, M.A.P.; MORUZZI, R.B.; PATRIZZI, L.J. Flotação por ar dissolvido aplicada na clarificação de águas que apresentam picos de turbidez. In: CONGRESSO BRASILEIRO DE ENGENHARIA SANITÁRIA E AMBIENTAL, 22., 2003, Joinville. Anais... Joinville: ABES, 2003. p. 1-9.

REN, P.; NAN, J.; ZHANG, X.; ZHENG, K. (2017) Analysis of floc morphology in a continuous-flow flocculation and sedimentation reactor. Journal of Environmental Sciences, v. 52, p. 268-275. https:// doi.org/10.1016/j.jes.2016.04.007

SUN, J.; QIN, L.; LI, G.; KANG, Y. (2013) Effect of hydraulic conditions on flocculation performances and floc characteristics in Chinese herbal extracts by chitosan and chitosan hydrochloride. Chemical Engineering Journal, v. 225, p. 641-649. https://doi.org/10.1016/j. cej.2013.03.108

VANDAMME, D.; MUYLAERT, K.; FRAEYE, I.; FOUBERT, I. (2014) Floc characteristics of Chlorella vulgaris: Influence of flocculation mode and presence of organic matter. Bioresource Technology, v. 151, p. 383-387. https://doi.org/10.1016/j. biortech.2013.09.112

WANG, D.; WU, R.; JIANG, Y.; CHOW, C.W.K. (2011) Characterization of floc structure and strength: Role of changing shear rates under various coagulation mechanisms. Colloids and Surfaces A: Physico chemical and Engineering Aspects, v. 379, n. 1-3, p. 36-42. https://doi. org/10.1016/j.colsurfa.2010.11.048

XU, Y.; CHEN, T.; LIU, Z.; ZHU, S.; CUI, F.; SHI, W. (2016) The impact of recycling alum-humic-floc (AHF) on the removal of natural organic materials (NOM): Behavior of coagulation and adsorption. Chemical Engineering Journal, v. 284, p. 1049-1057. https://doi. org/10.1016/j.cej.2015.09.069

YAO, M.; NAN, J.; CHEN, T. (2O14) Effect of particle size distribution on turbidity under various water quality levels during flocculation processes. Desalination, v. 354, p. 116-124. https://doi.org/10.1016/j. desal.2014.09.029

YUHENG, W.; SHENGGUANG, Z.; NA, L.; YIXIN, Y. (2O11) Influences of various aluminum coagulants on algae floc structure, strength and flotation effect. Procedia Environmental Sciences, v. 8, p. 75-80. https://doi.org/10.1016/j.proenv.2011.10.014

YUKSELEN, M.A.; GREGORY, J. (2004) The reversibility of flocs breakage. International Journal of Mineral Processing, v. 73, n. 2-4, p. 251-259. https://doi.org/10.1016/S0301-7516(03)00077-2 